\title{
Multivariate Autocontours for Specification Testing in Multivariate GARCH Models*
}

\author{
Gloria González-Rivera \\ Department of Economics \\ University of California, Riverside \\ gloria.gonzalez@ucr.edu
}

\author{
Emre Yoldas \\ Department of Economics \\ University of California, Riverside \\ emre.yoldas@email.ucr.edu
}

June 2008

\begin{abstract}
In this paper we propose a new battery of test statistics for dynamic specification and density functional form in a wide range of multivariate time series models including linear and non-linear VAR specifications with multivariate GARCH disturbances. The tests are applied to the vector of generalized errors that must be i.i.d. with a certain parametric multivariate probability density function under the null hypothesis of correct specification. The basic idea of the proposed methodology is to calculate the percentage of observations contained within the probability autocontour plots corresponding to the assumed multivariate density of the vector of independent innovations, and compare it to the population percentage. We develop $t$-tests based on a single autocontour and also more powerful chi-squared tests based on multiple autocontours. In the spirit of goodness-of-fit tests, we also propose an additional test that focuses on the multivariate density functional form of the vector of innovations. We explicitly consider parameter uncertainty and show that a simple bootstrap procedure overcomes this problem. We perform Monte-Carlo simulations to investigate the size and power properties of the test statistics in finite samples. We apply our tests to multivariate GARCH models fitted to excess returns on portfolios sorted according to market capitalization.
\end{abstract}

JEL Classification: C12, C15, C16, C22.

Keywords: Probability Contour Plot, Autocontour, Specification Test, Parameter Uncertainty, Bootstrap.

\footnotetext{
* We are grateful to Tim Bollerslev and an anonymous referee for helpful comments that significantly improved the presentation of the paper.
} 


\section{INTRODUCTION}

Even though there is an extensive literature on specification tests for univariate time series models, the development of new tests for multivariate models has been very slow. As an example, in the ARCH literature we have numerous univariate specifications for which we routinely scrutinize the standardized residuals for possible neglected dependence and deviation from the assumed conditional density. However, for multivariate GARCH models we rarely test for the assumed multivariate density and for cross-dependence in the residuals. Given the inherent difficulty of estimating multivariate GARCH models, the issue of dynamic misspecification at the system level -as important as it may be- seems to be secondary. Though univariate specification tests can be performed in each equation of the system, these tests are not independent from each other, and an evaluation of the system will demand adjustments in the size of any joint test that combines the results of the equation-by-equation univariate tests. Bauwens, Laurent, and Rombouts (2006) survey the latest developments in multivariate GARCH models and they also acknowledge the need for further research on multivariate diagnostic tests. There are some portmanteau statistics for neglected multivariate conditional heteroskedasticity as in Ling and Li (1997), Tse and Tsui (1999), and Duchesne and Lalancette (2003). Some of these tests have unknown asymptotic distributions when applied to the generalized GARCH residuals. Tse (2002)

proposes another type of misspecification test that is based on regressions of the standardized residuals on some explanatory variables. In that case, the usual OLS asymptotics do not apply, but it is possible to construct some statistics that are asymptotically chi-squared distributed under the null of no dynamic misspecification. None of these tests are concerned with the specification of the multivariate density. However, the knowledge of the density functional form is of paramount importance for density forecast evaluation, which is needed to assess the overall adequacy of the model. Recently, Bai and Chen (2008) adopted the empirical process based testing approach of Bai (2003), which is developed in the univariate framework, to multivariate models. They use single-indexed empirical processes to make computation feasible, but this causes loss of full consistency. Kalliovirta (2007) also takes an empirical process based approach and 
proposes several test statistics for checking dynamic misspecification and density functional form.

We propose a new battery of tests for dynamic specification and density functional form in multivariate time series models. We focus on the most popular models for which all the time dependence is confined to the first and second moments of the multivariate process. Multivariate dynamics in moments further than the second are difficult to find in the data and, to our knowledge, there are only a few attempts in the literature restricted to upmost bivariate systems. Our approach is not based on empirical processes, so we do not require probability integral transformations as opposed to the above mentioned studies testing for density specification. This makes dealing with parameter uncertainty relatively less challenging on theoretical grounds. When parameter estimation is required, we will adopt a quasi-maximum likelihood procedure as opposed to strict maximum likelihood, which assumes the knowledge of the true multivariate density. If the true density were known, it would be possible to construct tests for dynamic misspecification based on the martingale difference property of the score under the null. However, if the density function is unknown, a quasi-maximum likelihood estimator is the most desirable to avoid the inconsistency of the estimator that we would have obtained under a potentially false density function. The lack of consistency may also jeopardize the asymptotic distribution of the tests. Our approach is less demanding than any score-type testing in the sense that once quasi-maximum likelihood estimates are in place, we can proceed to test different proposals on the functional form of the conditional multivariate density function.

The proposed tests are based on the concept of "autocontour" introduced by GonzálezRivera, Senyuz, and Yoldas (2007) for univariate processes. Our methodology is applicable to a wide range of models including linear and non-linear VAR specifications with multivariate GARCH disturbances. The variable of interest is the vector of generalized innovations $\varepsilon_{t}=\left(\varepsilon_{1 t}, \varepsilon_{2 t}, \ldots, \varepsilon_{k t}\right)^{\prime}$ in a model $y_{t}=\mu_{t}\left(\theta_{01}\right)+H_{t}^{1 / 2}\left(\theta_{02}\right) \varepsilon_{t}$, where $y_{t}$ is a $k \times 1$ vector of variables with conditional mean vector $\mu_{t}$ and conditional covariance matrix $H_{t}$. Under the null hypothesis of correct dynamic specification the vector $\varepsilon_{t}$ must be i.i.d. with a certain parametric multivariate probability density function 
$f($.$) . Thus, if we consider the joint distribution of two vectors \varepsilon_{t}$ and $\varepsilon_{t-l}$, then under the null we have $f\left(\varepsilon_{t}, \varepsilon_{t-l}\right)=f\left(\varepsilon_{t}\right) f\left(\varepsilon_{t-l}\right)$. The basic idea of the proposed tests is to calculate the percentage of observations contained within the probability autocontour planes corresponding to the assumed multivariate density of the vector of independent innovations, i.e. $f\left(\varepsilon_{t}\right) f\left(\varepsilon_{t-l}\right)$, and to statistically compare it to the population percentage. We develop a battery of $t$-tests based on a single autocontour and also more powerful chisquared tests based on multiple autocontours, which have standard asymptotic distributions. Without parameter uncertainty the test statistics are all distribution free, but under parameter uncertainty there are nuisance parameters affecting the asymptotic distributions. We show that a simple bootstrap procedure overcomes this problem and yields the correct size even for moderate sample sizes. We also investigate the power properties of the test statistics in finite samples.

Since the null is a joint hypothesis, the rejection of the null begs the question on what is at fault. Thus, it is desirable to separate i.i.d-ness from density function. In the spirit of goodness-of-fit tests, we also propose an additional test that focuses on the multivariate density functional form of the vector of innovations. Following a similar approach, we construct the probability contours corresponding to the hypothesized multivariate density, $f\left(\varepsilon_{t}\right)$, and compare the sample percentage of observations falling within the contour to the population percentage. The goodness-of-fit tests are also constructed as $t$-statistics and chisquared statistics with standard distributions.

The organization of the paper is as follows. In Section 2, we describe the battery of tests, which follow from González-Rivera, Senyuz, Yoldas (2007), and the construction of the multivariate contours and autocontours. In Section 3, we offer some Monte Carlo simulation to assess the size and power of the tests in finite samples. In Section 4, we apply the tests to the generalized residuals of GARCH models with hypothesized multivariate Normal and multivariate Student-t innovations fitted to excess returns on five size portfolios. In Section 5, we conclude. 


\section{TESTING METHODOLOGY}

\subsection{Test Statistics}

Let $y_{t}=\left(y_{1 t}, \ldots, y_{k t}\right)$ and suppose that $y_{t}$ evolves according to the following process

$$
y_{t}=\mu_{t}\left(\theta_{01}\right)+H_{t}^{1 / 2}\left(\theta_{02}\right) \varepsilon_{t}, \quad t=1, \ldots, T
$$

where $\mu_{t}($.$) and H_{t}^{1 / 2}($.$) are both measurable with respect to time t-1$ sigma field, $\mathfrak{J}_{t-1}$, $H_{t}($.$) is positive definite, and \left\{\varepsilon_{t}\right\}$ is an i.i.d. vector process with zero mean and identity covariance matrix. The conditional mean vector, $\mu_{t}($.$) , and the conditional covariance$ matrix, $H_{t}($.$) , are fully parameterized by the parameter vector \theta_{0}=\left(\theta_{01}^{\prime}, \theta_{02}^{\prime}\right)^{\prime}$, which for now we assume to be known, but later on we will relax this assumption to account for parameter uncertainty.

If all the dependence is contained in the first and second conditional moments of the process $y_{t}$, then the null hypothesis of interest to test for model misspecification is

$$
H_{0}: \varepsilon_{t} \text { is i.i.d. with density } f(.) \text {. }
$$

The alternative hypothesis is the negation of the null. Though we wish to capture all the dynamic dependence of $y_{t}$ through the modeling of the conditional mean and conditional covariance matrix, there may be another degree of dependence that is built in the assumed multivariate density, $f($.$) . In fact, once we move beyond the assumption of multivariate$ normality, for instance when we assume a multivariate Student-t distribution, the components of the vector $\varepsilon_{t}$ are dependent among themselves and this information is only contained within the functional form of the density. This is why, among other reasons, it is of interest to incorporate the assumed density function in the null hypothesis.

Let us consider the joint distribution of two $k \times 1$ vectors $\varepsilon_{t}$ and $\varepsilon_{t-l}, l=1, \ldots, L<\infty$. Define a $2 k \times 1$ vector $\eta_{t}=\left(\varepsilon_{t}^{\prime}, \varepsilon_{t-l}^{\prime}\right)^{\prime}$ and let $\psi($.$) denote the associated density function.$ 
Under the null hypothesis of i.i.d. and correct probability density function, we can write $\psi\left(\eta_{t}\right)=f\left(\varepsilon_{t}\right) f\left(\varepsilon_{t-l}\right)$. Then, under the null, we define the $\alpha$-autocontour, $C_{l, \alpha}$, as the set of vectors $\left(\varepsilon_{t}^{\prime}, \varepsilon_{t-l}^{\prime}\right)$ that results from slicing the multivariate density, $\psi($.$) , at a certain$ value to guarantee that the set contains $\alpha \%$ of observations, that is,

$$
C_{l, \alpha}=\left\{S\left(\eta_{t}\right) \subset \mathfrak{R}^{2 k} \mid \int_{h_{1}}^{g_{1}} \cdots \int_{h_{2 k}}^{g_{2 k}} \psi\left(\eta_{t}\right) \mathrm{d} \eta_{1 t} \ldots \mathrm{d} \eta_{2 k, t} \leq \alpha\right\},
$$

where the limits of integration are determined by the density functional form so that the shape of the probability contours is preserved under integration, e.g. when the assumed density is normal, then the autocontours are $2 k$-spheres (a circle when $k=1$ ). We construct an indicator process defined as

$$
I_{t}^{l, \alpha}=\left\{\begin{array}{l}
1 \text { if } \eta_{t} \notin C_{l, \alpha} \\
0 \text { otherwise }
\end{array} .\right.
$$

The process $\left\{I_{t}^{l, \alpha}\right\}$ forms the building block of the proposed test statistics. Let $p_{\alpha} \equiv 1-\alpha$. Since the indicator is a Bernoulli random variable, its mean and variance are given by $E\left[I_{t}^{l, \alpha}\right]=p_{\alpha}$ and $\operatorname{Var}\left(I_{t}^{l, \alpha}\right)=p_{\alpha}\left(1-p_{\alpha}\right)$. Although $\left\{\varepsilon_{t}\right\}$ is an i.i.d. process, $\left\{I_{t}^{l, \alpha}\right\}$ exhibits some linear dependence because $I_{t}^{l, \alpha}$ and $I_{t-l}^{l, \alpha}$ share common information contained in $\varepsilon_{t-l}$. Hence, the autocovariance function of $\left\{I_{t}^{l, \alpha}\right\}$ is given by

$$
\gamma_{h}^{\alpha}=\left\{\begin{array}{l}
P\left(I_{t}^{l, \alpha}=1, I_{t-h}^{l, \alpha}=1\right)-p_{\alpha}^{2} \text { if } h=l \\
0 \text { otherwise }
\end{array} .\right.
$$


Proposition 1. Define $\hat{p}_{\alpha}^{l}=(T-l)^{-1} \sum_{t=1}^{T-l} I_{t}^{l, \alpha}$. Under the null hypothesis,

$$
t_{l, \alpha}=\frac{\sqrt{T-l}\left(\hat{p}_{\alpha}^{l}-p_{\alpha}\right)}{\sigma_{l, \alpha}} \rightarrow_{d} \mathrm{~N}(0,1),
$$

where $\sigma_{l, \alpha}^{2}=p_{\alpha}\left(1-p_{\alpha}\right)+2 \gamma_{l}^{\alpha}$.

Proof: See González-Rivera, Senyuz, and Yoldas (2007) for all mathematical proofs.

Now let us consider a finite number of contours, $\left(\alpha_{1}, \ldots, \alpha_{n}\right)$, jointly. Let $p_{\alpha}=\left(p_{\alpha_{1}}, \ldots, p_{\alpha_{n}}\right)^{\prime} \quad$ where $\quad p_{\alpha_{i}}=1-\alpha_{i}$, and define $\hat{p}_{\alpha_{i}}^{l}=(T-l)^{-1} \sum_{t=1}^{T-l} I_{t}^{l, \alpha_{i}} \quad$ for $i=1, \ldots, n$. We then collect all the $\hat{p}_{\alpha_{i}}^{l}$ 's in a $n \times 1$ vector, $\hat{p}_{\alpha}^{l}=\left(\hat{p}_{1}, \ldots, \hat{p}_{n}\right)^{\prime}$.

Proposition 2: Under the null hypothesis,

$$
\sqrt{T-l}\left(\hat{p}_{\alpha}^{l}-p_{\alpha}\right) \rightarrow_{d} \mathrm{~N}(0, \Xi)
$$

where the elements of $\Xi$ are $\xi_{i j}=\min \left(p_{\alpha_{i}}, p_{\alpha_{j}}\right)-p_{\alpha_{i}} p_{\alpha_{j}}+\operatorname{Cov}\left(I_{t}^{l, \alpha_{i}}, I_{t-l}^{l, \alpha_{j}}\right)$

$+\operatorname{Cov}\left(I_{t}^{l, \alpha_{j}}, I_{t-l}^{l, \alpha_{i}}\right)$. Then, it directly follows that

$$
J_{n}^{l}=(T-l)\left(\hat{p}_{\alpha}^{l}-p_{\alpha}\right)^{\prime} \Xi^{-1}\left(\hat{p}_{\alpha}^{l}-p_{\alpha}\right) \rightarrow_{d} \chi^{2}(n) .
$$

A complementary test to those described above can be constructed in the spirit of goodness-of-fit. Suppose that we consider only the vector $\varepsilon_{t}$ and we wish to test in the direction of density functional form. We construct the probability contour sets $C_{\alpha}$ 
corresponding to the probability density function that is assumed under the null hypothesis. The set is given by

$$
C_{\alpha}=\left\{S\left(\varepsilon_{t}\right) \subset \mathfrak{R}^{k} \mid \int_{h_{1}}^{g_{1}} \cdots \int_{h_{k}}^{g_{k}} f\left(\varepsilon_{t}\right) \mathrm{d} \varepsilon_{1 t} \ldots \mathrm{d} \varepsilon_{k t} \leq \alpha\right\}
$$

Then, as before, we construct an indicator process as follows

$$
I_{t}^{\alpha}=\left\{\begin{array}{l}
1 \text { if } \varepsilon_{t} \notin C_{\alpha} \\
0 \text { otherwise }
\end{array}\right.
$$

for which the mean and variance are $E\left[I_{t}^{\alpha}\right]=1-\alpha$ and $\operatorname{Var}\left(I_{t}^{\alpha}\right)=\alpha(1-\alpha)$, respectively. The main difference between the sets $C_{l, \alpha}$ and $C_{\alpha}$ is that the latter does not explicitly consider the time-independence assumed under the null and, therefore, the following tests based on $C_{\alpha}$ will be less powerful against independence. There is also a difference in the properties of the indicator process. Now, the indicator is also an i.i.d. process, and the analogous tests to those of Propositions 1 and 2 will have a simpler asymptotic distribution.

Let $p_{\alpha}=1-\alpha$ and define an estimator of $p_{\alpha}$ as $\tilde{p}_{\alpha}=T^{-1} \sum_{t=1}^{T} I_{t}^{\alpha}$. Under the null hypothesis the distribution of the analogue test statistic to that of Proposition 1 is

$$
t_{\alpha}=\frac{\sqrt{T}\left(\tilde{p}_{\alpha}-p_{\alpha}\right)}{p_{\alpha}\left(1-p_{\alpha}\right)} \rightarrow_{d} \mathrm{~N}(0,1) .
$$

If, as in Proposition 2, now we jointly consider a finite number of contours and define the vectors $\quad p_{\alpha}=\left(p_{\alpha_{1}}, \ldots, p_{\alpha_{n}}\right)^{\prime} \quad$ and $\quad \tilde{p}_{\alpha}=\left(\tilde{p}_{\alpha_{1}}, \ldots, \tilde{p}_{\alpha_{n}}\right)^{\prime}$, where $\quad p_{\alpha_{i}}=1-\alpha_{i} \quad$ and 
$\widetilde{p}_{\alpha_{i}}=T^{-1} \sum_{t=1}^{T} I_{t}^{\alpha_{i}}$. Then $\sqrt{T}\left(\widetilde{p}_{\alpha}-p_{\alpha}\right) \rightarrow_{d} \mathrm{~N}(0, \Xi)$ where the elements of $\Xi$ simplify to $\xi_{i j}=\min \left(p_{\alpha_{i}}, p_{\alpha_{j}}\right)-p_{\alpha_{i}} p_{\alpha_{j}}$ and, it follows that

$$
\widetilde{J}_{n}=T\left(\widetilde{p}_{\alpha}-p_{\alpha}\right)^{\prime} \Xi^{-1}\left(\widetilde{p}_{\alpha}-p_{\alpha}\right) \rightarrow_{d} \chi^{2}(n)
$$

Note that to make these tests operational we replace the covariance terms by their sample counterparts. Furthermore, the asympotic normality results established above still hold under parameter uncertainty as shown by González-Rivera, Senyuz, and Yoldas (2007). However, one needs to deal with nuisance parameters in the asymptotic covariance matrices to make the statistics operational. They suggest using a parametric bootstrap procedure, which imposes all restrictions of the null hypothesis to estimate asymptotic covariance matrices under parameter uncertainty. Specifically, after the model is estimated, bootstrap samples are generated by using the estimated model as the data generating process where innovation vectors are drawn from the hypothesized parametric distribution. Their Monte-Carlo simulations indicate that this approach provides satisfactory results. Hence, in this paper we take the same approach in our applications.

\subsection{Multivariate Contours and Autocontours}

\section{Multivariate Normal Distribution}

In this case the density function is $f\left(\varepsilon_{t}\right)=(2 \pi)^{-k / 2} \exp \left(-0.5 \varepsilon_{t}^{\prime} \varepsilon_{t}\right)$. Let $\bar{f}_{\alpha}$ denote the value of the density such that the corresponding probability contour contains $\alpha \%$ of the observations. Then the equation describing this contour is

$$
q_{\alpha}=\varepsilon_{t}^{\prime} \varepsilon_{t} \equiv \varepsilon_{1 t}^{2}+\varepsilon_{2 t}^{2}+\cdots+\varepsilon_{k t}^{2},
$$

where $q_{\alpha}=-2 \ln \left(\bar{f}_{\alpha} \times(2 \pi)^{k / 2}\right)$. Hence, the $C_{\alpha}$ contour set is defined as follows 


$$
C_{\alpha}=\left\{S\left(\varepsilon_{t}\right) \subset \mathfrak{R}^{k} \mid \int_{-g_{1}}^{g_{1}} \ldots \int_{-g_{k}}^{g_{k}}(2 \pi)^{-k / 2} \exp \left(-0.5 \varepsilon_{t}^{\prime} \varepsilon_{t}\right) \mathrm{d} \varepsilon_{1 t} \ldots \mathrm{d} \varepsilon_{k t} \leq \alpha\right\}
$$

where $g_{1}=\sqrt{q_{\lambda}}, g_{i}=\sqrt{q_{\lambda}-\sum_{j=1}^{i-1} \varepsilon_{j t}^{2}}$ for $i=2, \ldots, k$, and $\lambda \leq \alpha$. We need to determine the mapping $q_{\alpha}$ in order to construct the indicator process. Let $x_{t}=\varepsilon_{t}^{\prime} \varepsilon_{t}$, then $x_{t} \sim \chi^{2}(k)$ and we have $q_{\alpha} \equiv \inf \left\{q: F_{x_{t}}(q) \geq \alpha\right\}$, where $F_{x_{t}}$ is the cumulative distribution function of a chi-squared random variable with $k$ degrees of freedom. As a result, the indicator series is obtained as follows

$$
I_{t}^{\alpha}=\left\{\begin{array}{l}
1 \text { if } \varepsilon_{t}^{\prime} \varepsilon_{t}>q_{\alpha} \\
0 \text { otherwise }
\end{array} .\right.
$$

To construct the autocontour $C_{l, \alpha}$, we consider the joint distribution of $\varepsilon_{t}$ and $\varepsilon_{t-l}$. Let $\eta_{t}=\left(\varepsilon_{t}^{\prime}, \varepsilon_{t-l}^{\prime}\right)^{\prime}$, then the density of interest is given by $\psi\left(\eta_{t}\right)=(2 \pi)^{-k} \exp \left(-0.5 \eta_{t}^{\prime} \eta_{t}\right)$. Hence, the autocontour equation is given by

$$
d_{\alpha}=\eta_{t}^{\prime} \eta_{t} \equiv \eta_{1 t}^{2}+\cdots+\eta_{2 k, t}^{2}
$$

where $d_{\alpha}=-2 \ln \left(\bar{\psi}_{\alpha} \times(2 \pi)^{k}\right)$. Following the same arguments as above, the corresponding indicator process is

$$
I_{t}^{l, \alpha}=\left\{\begin{array}{l}
1 \text { if } \eta_{t}^{\prime} \eta_{t}>d_{\alpha} \\
0 \text { otherwise }
\end{array}\right.
$$

where $d_{\alpha} \equiv \inf \left\{d: F_{x_{t}}(d) \geq \alpha\right\}, x_{t}=\eta_{t}^{\prime} \eta_{t}$, and $F_{x_{t}}$ is the cumulative distribution function of a chi-squared random variable with $2 k$ degrees of freedom. 


\section{Student-t Distribution}

The multivariate density function is

$$
f\left(\varepsilon_{t}\right)=G(k, v)\left[1+\varepsilon_{t}^{\prime} \varepsilon_{t} /(v-2)\right]^{-(k+v) / 2},
$$

where $G(k, v)=\Gamma[(v+k) / 2] /\left\{[\pi(v-2)]^{0.5 k} \Gamma(v / 2)\right\}$. Then the equation for the $\alpha-$ probability contour is

$$
q_{\alpha}=1+\varepsilon_{t}^{\prime} \varepsilon_{t} /(v-2)
$$

where $q_{\alpha}=\left[\bar{f}_{\alpha} / G(k, v)\right]^{(k+v) / 2}$. As a result, the $C_{\alpha}$ contour set is defined as

$$
C_{\alpha}=\left\{S\left(\varepsilon_{t}\right) \subset \mathfrak{R}^{k} \mid \int_{-g_{1}}^{g_{1}} \ldots \int_{-g_{k}}^{g_{k}} G(k, v)\left(1+\varepsilon_{t}^{\prime} \varepsilon_{t} /(v-2)\right) \mathrm{d} \varepsilon_{1 t} \ldots \mathrm{d} \varepsilon_{k t} \leq \alpha\right\}
$$

where $g_{1}=\sqrt{\left(q_{\lambda}-1\right)(v-2)}, g_{i}=\sqrt{\left(q_{\lambda}-1\right)(v-2)-\sum_{j=1}^{i-1} \varepsilon_{j t}^{2}}$ for $i=2, \ldots, k$, and $\lambda \leq \alpha$. Now let $x_{t}=1+\varepsilon_{t}^{\prime} \varepsilon_{t} /(v-2)$, then $x_{t} \equiv 1+(k / v) w_{t}$ where $w_{t}$ has an F-distribution with $(k, v)$ degrees of freedom. Consequently, we have $q_{\alpha} \equiv \inf \left\{q: F_{w_{t}}[v(q-1) / k] \geq \alpha\right\}$. Then the indicator series is defined as

$$
I_{t}^{\alpha}=\left\{\begin{array}{l}
1 \text { if } 1+\varepsilon_{t}^{\prime} \varepsilon_{t} /(v-2)>q_{\alpha} \\
0 \text { otherwise }
\end{array}\right.
$$

To construct the autocontour $C_{l, \alpha}$, we consider the joint distribution of $\varepsilon_{t}$ and $\varepsilon_{t-l}$ under the null hypothesis, which is

$$
\psi\left(\varepsilon_{t}, \varepsilon_{t-l}\right)=G(k, v)^{2}\left[\left(1+\varepsilon_{t}^{\prime} \varepsilon_{t} /(v-2)\right)\left(1+\varepsilon_{t-l}^{\prime} \varepsilon_{t-l} /(v-2)\right)\right]^{-(k+v) / 2} .
$$


Then, the equation for the $\alpha$-probability autocontour is given by

$$
d_{\alpha}=1+\left(\varepsilon_{t}^{\prime} \varepsilon_{t}+\varepsilon_{t-l}^{\prime} \varepsilon_{t-l}\right) /(v-2)+\left(\varepsilon_{t}^{\prime} \varepsilon_{t}\right)\left(\varepsilon_{t-l}^{\prime} \varepsilon_{t-l}\right) /(v-2)^{2}
$$

Let $x_{t}=1+\left(\varepsilon_{t}^{\prime} \varepsilon_{t}+\varepsilon_{t-l}^{\prime} \varepsilon_{t-l}\right) /(v-2)+\left(\varepsilon_{t}^{\prime} \varepsilon_{t}\right)\left(\varepsilon_{t-l}^{\prime} \varepsilon_{t-l}\right) /(v-2)^{2}$, then we have $x_{t}=1+(k / v)$ $\times\left[\left(w_{1 t}+w_{2 t}\right)+(k / v)\left(w_{1 t} w_{2 t}\right)\right]$ where $w_{1 t}$ and $w_{2 t}$ are independent random variables with an F-distribution with $(k, v)$ degrees of freedom. Similar to the previous case, we have $d_{\alpha} \equiv \inf \left\{d: F_{x_{t}}(d) \geq \alpha\right\}$, but we do not have readily available results for the quantiles of $x_{t}$ as before. A plausible solution is using Monte-Carlo simulation to approximate the quantiles of interest as we already know that $x_{t}$ is a specific function of two independent F-distributed random variables.

As an illustration, we provide sample contour and autocontour plots under normal and Student-t (with $v=5$ ) distributions in Figure-1. Due to the graphical constraints imposed by high dimensionality, we consider $k=2$ and $k=1$ for $C_{\alpha}$ and $C_{l, \alpha}$ respectively. Note that while $C_{\alpha}$ and $C_{l, \alpha}$ are of identical shape under normality, since the product of two independent normal densities yields a bivariate normal density, this is not the case under the Student-t distribution.

Figure-1: Contour and Autocontour Plots under Normal and Student-t Distributions $C_{\alpha}$ under bivariate Normal and Student-t Distributions $\alpha \in\{0.5,0.7,0.9,0.99\}$
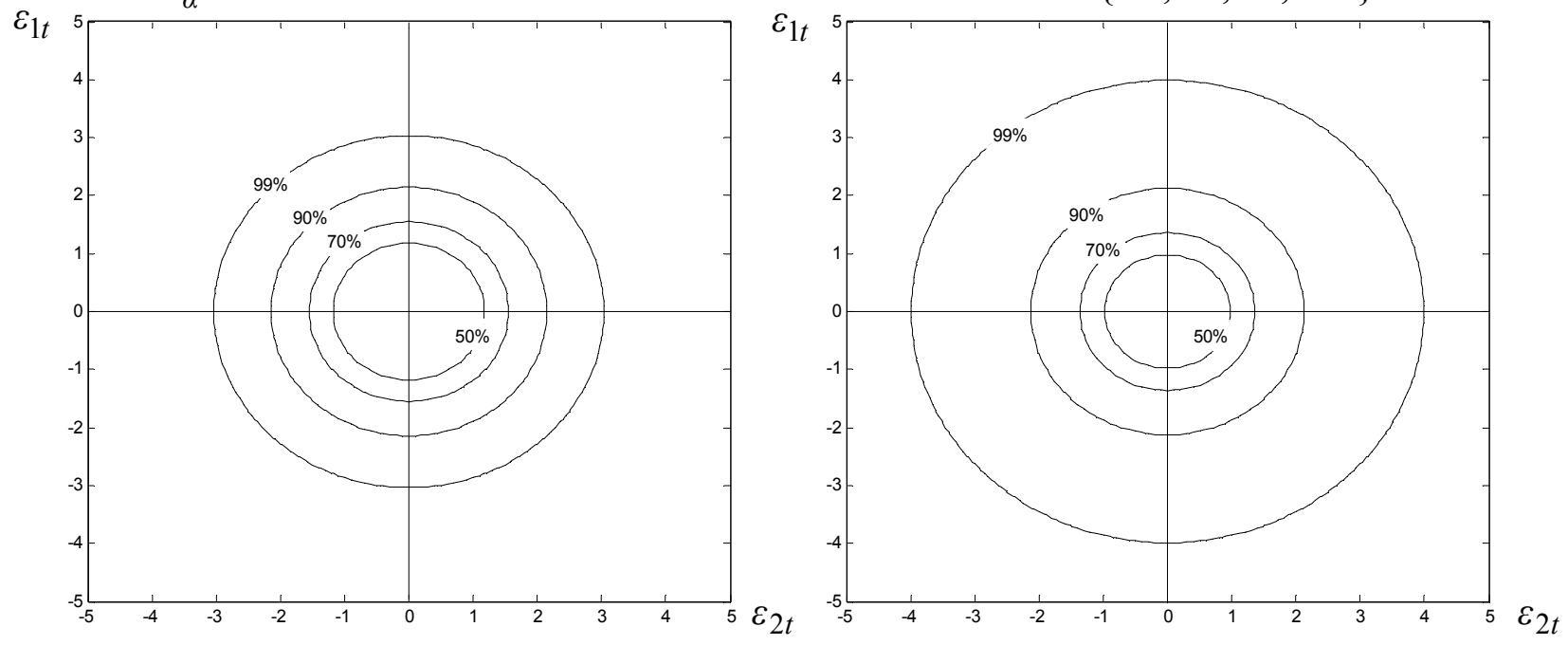


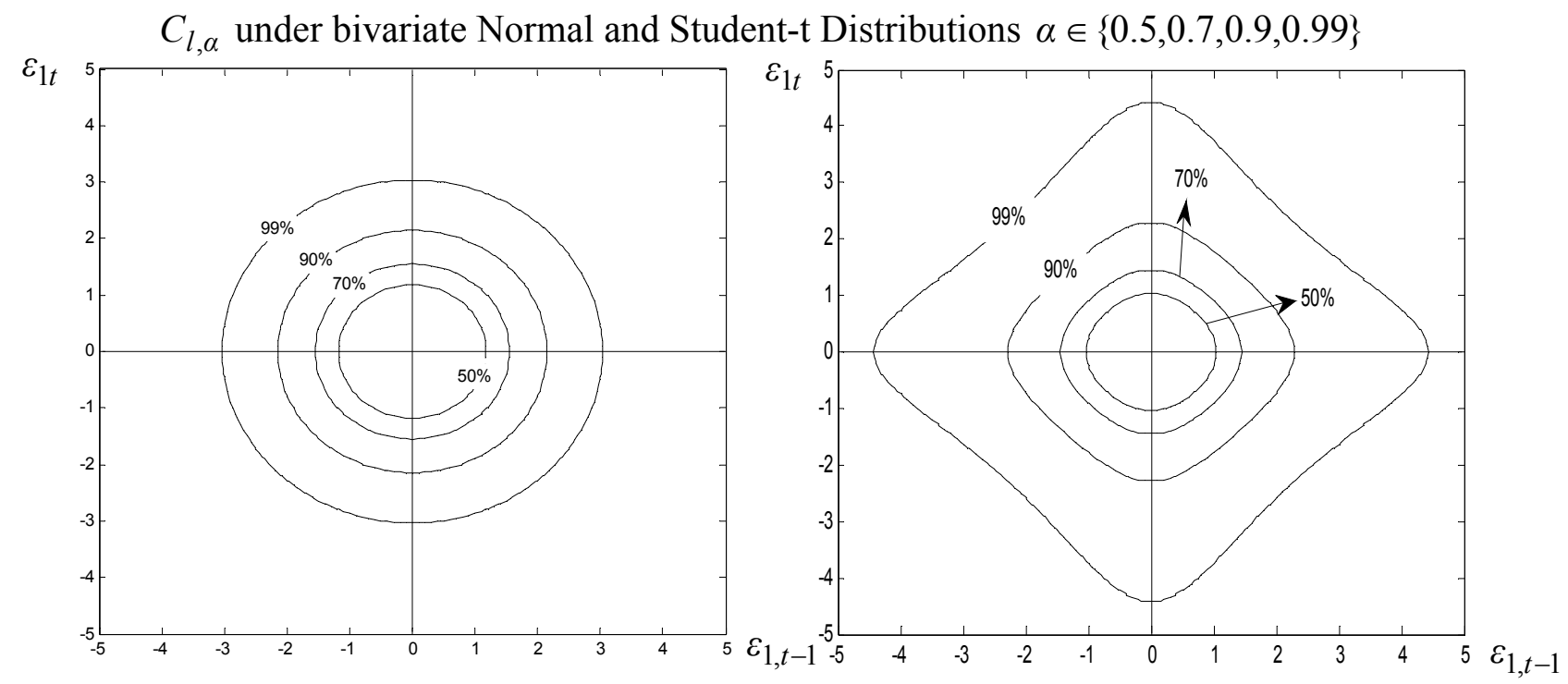

\section{MONTE-CARLO SIMULATIONS}

We investigate the size and power properties of the proposed tests in finite samples by Monte Carlo simulations for two cases: when the parameters of the model are known and when they are unknown and need to be estimated.

\subsection{Size Simulations}

For the size experiments we consider two alternative distributions for the innovation process: a multivariate Normal, $\varepsilon_{t} \sim$ i.i.d. $\mathrm{N}\left(0, \mathrm{I}_{k}\right)$, and a multivariate Student-t with 5 degrees of freedom, $\varepsilon_{t} \sim$ i.i.d.t $\left(0, \mathrm{I}_{k}, 5\right)$. Under parameter uncertainty, we consider a simple multivariate location-scale model: $y_{t}=\mu+H^{1 / 2} \varepsilon_{t}$ where we set $\mu=0$ and $H=I_{k}$. We consider both distributions under parameter uncertainty and apply the tests to the estimated standardized residual vector, $\hat{\varepsilon}_{t}=\hat{H}^{-1 / 2}\left(y_{t}-\hat{\mu}\right)$, where we obtain $H^{1 / 2}$ by using the Cholesky decomposition ${ }^{1}$. The asymptotic variance of the tests is obtained by the simple parametric bootstrap procedure outlined above (see Section 2.1). The number of Monte Carlo replications is equal to 1000 , and the number of bootstrap replications is set

\footnotetext{
${ }^{1}$ Alternative decompositions can be used to calculate the square-root matrix. We conjecture that the choice of the decomposition technique is not critical for application of our tests.
} 
to 500. We consider 13 autocontours $(n=13)$ with coverage levels $(\%): 1,5,10,20,30$, $40,50,60,70,80,90,95$, and 99, spanning the entire density function ${ }^{2}$. We start with a sample size of 250 and consider increments of 250 up to 2,000 observations. In all experiments, the nominal size is $5 \%$.

In Tables $1 \mathrm{a}$ and $1 \mathrm{~b}$ we present the simulated size results for the $J_{n}^{l}$-statistics. We consider a system of 2 equations $(k=2)$ and a system of 5 equations $(k=5)$. For a small sample of 250 observations, the $J_{n}^{l}$-statistics are oversized for both densities and both systems. However, under parameter uncertainty, the bootstrap procedure seems to correct to some extent the oversize behavior. For samples of 1000 and more observations, the simulated size is within an acceptable range of values. There are no major differences between the results for the small versus the large systems of equations indicating that the dimensionality of the system is not an issue for the implementation of these tests.

Table 1a: Size of the $J_{n}^{l}$-statistics

\begin{tabular}{ccccccccccccc}
\hline \hline$T$ & $J_{13}^{1}$ & $J_{13}^{2}$ & $J_{13}^{3}$ & $J_{13}^{4}$ & $J_{13}^{5}$ & $J_{13}^{1}$ & $J_{13}^{2}$ & $J_{13}^{3}$ & $J_{13}^{4}$ & $J_{13}^{5}$ \\
\hline \multicolumn{1}{c}{ Panel a: Normal $(k=2)$} & \multicolumn{6}{c}{ Panel b: Student-t $(k=2)$} \\
\hline 250 & 11.3 & 11.3 & 11.6 & 8.8 & 11.8 & 10.5 & 11.0 & 10.5 & 12.3 & 9.4 \\
500 & 6.5 & 6.0 & 5.8 & 5.9 & 8.0 & 7.5 & 5.8 & 5.9 & 7.0 & 6.2 \\
1000 & 6.8 & 5.0 & 6.2 & 5.3 & 4.9 & 7.2 & 5.2 & 5.1 & 5.4 & 6.0 \\
2000 & 6.4 & 5.1 & 5.7 & 4.1 & 4.8 & 7.2 & 5.8 & 5.5 & 6.4 & 6.4 \\
\hline \multicolumn{1}{c}{ Panel a: } & Normal $(k=5)$ & \multicolumn{1}{c}{ Panel b: Student-t $(k=5)$} \\
\hline 250 & 12.7 & 11.8 & 11.5 & 14.0 & 12.9 & 10.4 & 11.7 & 12.3 & 10.3 & 11.6 \\
500 & 9.2 & 8.4 & 6.9 & 7.6 & 8.3 & 7.3 & 6.6 & 7.3 & 7.9 & 8.1 \\
1000 & 6.3 & 7.1 & 5.5 & 6.0 & 6.4 & 5.9 & 4.8 & 6.6 & 5.7 & 7.8 \\
2000 & 5.3 & 5.6 & 5.3 & 3.4 & 6.5 & 6.9 & 4.8 & 5.7 & 5.5 & 5.4 \\
\hline \hline
\end{tabular}

\footnotetext{
${ }^{2}$ Our choice of the contour coverage levels is motivated by the need of covering the entire range of the density, from the tails to the very center as we do not have a theoretical result indicating the optimal choice of the number of contours to guide our practice. The flexibility of our approach permits considering different types of coverage levels depending on the purpose of application, e.g. concentrating on tails for risk models. Note also that the Monte-Carlo results presented below provide guidance as to how far one can go in the tails and the center of the denisty without losing precision in finite samples. Additional Monte-Carlo simulations, not reported here to save space, also indicate that the size and power results are robust to the number of contours as long as the range considered is identical, i.e. a finer grid does not change the results.
} 
Table 1b: Size of the $J_{n}^{l}$-statistics under Parameter Uncertainty

\begin{tabular}{cccccccccccc}
\hline \hline$T$ & $J_{13}^{1}$ & $J_{13}^{2}$ & $J_{13}^{3}$ & $J_{13}^{4}$ & $J_{13}^{5}$ & & $J_{13}^{1}$ & $J_{13}^{2}$ & $J_{13}^{3}$ & $J_{13}^{4}$ & $J_{13}^{5}$ \\
\hline \multicolumn{4}{c}{ Panel a: } & Normal $(k=2)$ & \multicolumn{6}{c}{ Panel b: Student-t $(k=2)$} \\
\hline 250 & 8.1 & 6.1 & 7.3 & 7.5 & 6.9 & 6.8 & 6.4 & 7.8 & 6.5 & 6.0 \\
500 & 7.5 & 5.9 & 5.8 & 7.3 & 7.4 & & 7.5 & 6.7 & 8.3 & 8.0 & 8.1 \\
1000 & 8.1 & 5.8 & 8.0 & 7.3 & 6.6 & 8.5 & 6.9 & 8.8 & 8.3 & 7.6 \\
2000 & 5.7 & 5.4 & 7.7 & 6.4 & 4.8 & 6.2 & 7.6 & 7.6 & 6.4 & 7.0 \\
\hline \multicolumn{4}{c}{ Panel a: } & Normal $(k=5)$ & \multicolumn{6}{c}{ Panel b: Student-t $(k=5)$} \\
\hline 250 & 10.5 & 9.3 & 7.7 & 9.2 & 8.1 & 7.1 & 7.3 & 6.3 & 7.2 & 6.3 \\
500 & 7.7 & 6.9 & 6.3 & 6.9 & 7.6 & 6.8 & 5.5 & 6.0 & 6.9 & 6.4 \\
1000 & 5.9 & 6.1 & 7.1 & 5.5 & 5.5 & 6.4 & 5.7 & 6.8 & 7.5 & 6.6 \\
2000 & 8.0 & 8.0 & 7.4 & 6.8 & 7.1 & 7.0 & 6.5 & 7.3 & 6.3 & 7.9 \\
\hline \hline
\end{tabular}

In Tables $2 \mathrm{a}$ and $2 \mathrm{~b}$ we show the simulated size for the $\widetilde{J}_{n}$-statistics, which should be understood primarily as goodness-of-fit tests as they do not explicitly take into account the independence of the innovations over time. The sizes reported in Table $2 \mathrm{a}$ are very good, though those in Table $2 \mathrm{~b}$ tend to be slightly larger than $5 \%$ mainly for small samples. However, when we consider the tests with individual contours (see Table 3 below), the size distortion tends to disappear.

Table 2a: Size of the $\widetilde{J}_{n}$-statistics $(n=13)$

\begin{tabular}{ccccc}
\hline \hline & \multicolumn{2}{c}{ Normal } & \multicolumn{2}{c}{ Student-t } \\
$T$ & $k=2$ & $k=5$ & $k=2$ & $k=5$ \\
\hline 250 & 5.7 & 6.3 & 4.3 & 6.6 \\
500 & 4.9 & 5.3 & 3.1 & 5.1 \\
1000 & 5.7 & 5.7 & 5.6 & 5.3 \\
2000 & 5.6 & 6.2 & 4.9 & 5.6 \\
\hline \hline
\end{tabular}


Table 2b: Size of the $\widetilde{J}_{n}$-statistics $(n=13)$ under Parameter Uncertainty

\begin{tabular}{ccccc}
\hline \hline & \multicolumn{2}{c}{ Normal } & \multicolumn{2}{c}{ Student-t } \\
$T$ & $k=2$ & $k=5$ & $k=2$ & $k=5$ \\
\hline 250 & 6.9 & 9.1 & 7.3 & 6.8 \\
500 & 7.0 & 6.1 & 6.8 & 6.7 \\
1000 & 6.7 & 5.5 & 6.7 & 5.6 \\
2000 & 6.4 & 7.4 & 6.8 & 5.7 \\
\hline \hline
\end{tabular}

For the $t$-tests, which are based on individual contours, the simulated sizes are very good. In Table 3, we report these results for the case of parameter uncertainty. The major size distortions occur for small samples at the extreme contour $t_{13}(99 \%$ coverage), but this is not very surprising since we do not expect enough variation in the indicator series for small samples.

Table 3: Size of the t-statistics under Parameter Uncertainty

\begin{tabular}{|c|c|c|c|c|c|c|c|c|c|c|c|c|c|}
\hline$T$ & $t_{1}$ & $t_{2}$ & $t_{3}$ & $t_{4}$ & $t_{5}$ & $t_{6}$ & $t_{7}$ & $t_{8}$ & $t_{9}$ & $t_{10}$ & $t_{11}$ & $t_{12}$ & $t_{13}$ \\
\hline & \multicolumn{13}{|c|}{ Panel a: Normal $(k=2)$} \\
\hline 250 & 5.0 & 4.6 & 5.2 & 5.1 & 6.5 & 6.7 & 5.7 & 4.9 & 5.2 & 4.6 & .0 & 4.8 & 2.0 \\
\hline 500 & 4.3 & 4.2 & 5.3 & 5.4 & 4.1 & 4.6 & 4.5 & 5.1 & 5.3 & 5.2 & .1 & 4.7 & 6.4 \\
\hline 1000 & 4.7 & 4.2 & 5.2 & 5.8 & 5.4 & 5.5 & 5.2 & 5.7 & 5.7 & 4.6 & .9 & 7.6 & 3.7 \\
\hline 00 & 5.4 & 3.9 & .1 & 4.0 & $J .0$ & 5.3 & 5.3 & 6.2 & 4.8 & 5.9 & 3 & 6.4 & 4.9 \\
\hline \multicolumn{14}{|c|}{ Panel b: Normal $(k=5)$} \\
\hline 250 & 4.5 & 6.2 & 5.3 & 5.0 & 4.5 & 5.2 & 5.3 & 5.8 & 5.5 & 5.1 & 1 & 6.7 & 2.1 \\
\hline 500 & 4.1 & 4.8 & 5.8 & 4.8 & 6.0 & 5.6 & 5.3 & 6.4 & 6.5 & 4.3 & 6.3 & 6.0 & 6.3 \\
\hline 000 & 3.8 & 5.3 & 5.7 & 5.3 & 4.9 & 5.2 & 3.8 & 3.3 & 4.6 & 5.3 & 6.0 & 4.7 & 3.9 \\
\hline 2000 & 4.5 & 5.3 & 5.0 & 5.0 & 4.6 & 4.1 & 5.4 & 6.0 & 4.6 & 5 & $\underline{5}$ & 4.4 & 6.5 \\
\hline \multicolumn{14}{|c|}{ Panel c: Student-t $(k=2)$} \\
\hline 250 & 4.5 & 5.1 & 5.3 & 4.9 & 4.9 & 6.0 & 4.8 & 4.6 & 4.5 & 5.4 & 5.7 & 4.3 & 8.7 \\
\hline 500 & 4.5 & 6.1 & 5.9 & 4.8 & 4.5 & 4.2 & 4.9 & 5.3 & 4.2 & 5.3 & 6.1 & 5.9 & 4.9 \\
\hline 000 & 4.3 & 5.9 & 6.4 & 5.8 & 5.7 & 5.5 & 6.6 & 6.4 & 5.9 & 5.8 & 5.5 & 6.0 & 6.3 \\
\hline 2000 & 5.7 & 5.0 & 5.2 & 5.4 & 5.5 & 4.7 & 5.4 & 5.9 & 5.5 & 5.0 & 4.9 & 5.2 & 4.8 \\
\hline \multicolumn{14}{|c|}{ Panel d: Student-t $(k=5)$} \\
\hline 250 & 4.5 & 5.5 & 4.8 & 4.6 & 5.8 & 6.0 & 7.6 & 6.7 & 7.0 & 6.6 & 5.8 & 4.1 & 8.4 \\
\hline 500 & 4.6 & 5.4 & 6.4 & 4.9 & 4.9 & 6.6 & 5.8 & 7.1 & 7.7 & 6.5 & 5.4 & 5.0 & 5.9 \\
\hline 1000 & 3.4 & 4.2 & 4.9 & 5.5 & 4.7 & 6.2 & 5.8 & 5.3 & 5.2 & 6.0 & 5.2 & 4.7 & 3.7 \\
\hline 2000 & 5.1 & 5.6 & 5.3 & 5.2 & 5.2 & 5.0 & 5.3 & 4.4 & 5.3 & 6.1 & 5.0 & 5.1 & 3.8 \\
\hline
\end{tabular}




\subsection{Power simulations}

We investigate the power of the tests by generating data from a system with two equations that follows three different stochastic processes. We maintain the null hypothesis as $y_{t}=\mu+H^{1 / 2} \varepsilon_{t}$, where $\varepsilon_{t} \sim$ i.i.d. $\mathrm{N}\left(0, I_{k}\right)$, and consider the following DGP's:

DGP 1: $y_{t}=\mu+H^{1 / 2} \varepsilon_{t}$, where $\varepsilon_{t} \sim$ i.i.d.t $\left(0, \mathrm{I}_{2}, 5\right), \mu=0$, and $H=\mathrm{I}_{2}$. In this case, we maintain the independence hypothesis and analyze departures from the hypothesized density function by generating i.i.d. observations from a multivariate Student-t distribution with 5 degrees of freedom.

DGP 2: $y_{t}=A y_{t-1}+H^{1 / 2} \varepsilon_{t}$, where $\varepsilon_{t} \sim$ i.i.d. N $\left(0, \mathrm{I}_{2}\right), a_{11}=0.7, a_{12}=0.1, a_{21}=0.03$, $a_{22}=0.85$, and $H=\mathrm{I}_{2}$. In this case, we maintain the same density function as that of the null hypothesis and analyze departures from the independence assumption by considering a linear $\operatorname{VAR}(1)$.

DGP 3: $y_{t}=H_{t}^{1 / 2} \varepsilon_{t}, \quad \varepsilon_{t} \sim$ i.i.d. $\mathrm{N}\left(0, \mathrm{I}_{2}\right), \quad$ with $H_{t}=C+A^{\prime} y_{t-1} y_{t-1}^{\prime} A+G^{\prime} H_{t-1} G$ and parameter values $A=0.1^{1 / 2} \times \mathrm{I}_{2}, G=0.85^{1 / 2} \times \mathrm{I}_{2}$, and $C=V-A^{\prime} V A-G^{\prime} V G$ where $V$ is the unconditional covariance matrix with $v_{11}=v_{22}=1$ and $v_{12}=0.5$. In this case, we analyze departures from both independence and density functional form by generating data from a system with multivariate conditional heteroscedasticity.

In Table 4 we report the power of the $J_{n}^{l}$-statistic. The test is the most powerful to detect departures from density functional form (DGP 1) as the rejection rates are almost $100 \%$ even in small samples. For departures from independence, the test has better power to detect dependence in the conditional mean (DGP 2) than in the conditional variance (DGP 3). As expected, in the case of the VAR(1) model (DGP 2), the power decreases as $l$ becomes larger indicating first order linear dependence. The power is also very good (69\%) for small samples of 250 observations. In the case of the GARCH model (DGP 3), the rejection rate reaches $60 \%$ for sample sizes of 500 observations and above. 
Table 4: Power of the $J_{n}^{l}$-statistics under Parameter Uncertainty

\begin{tabular}{cccccc}
\hline \hline$T$ & $J_{13}^{1}$ & $J_{13}^{2}$ & $J_{13}^{3}$ & $J_{13}^{4}$ & $J_{13}^{5}$ \\
\hline \multicolumn{5}{c}{ Panel a: DGP 1 } \\
\hline 250 & 98.6 & 98.2 & 98.6 & 97.8 & 98.3 \\
500 & 100.0 & 100.0 & 100.0 & 100.0 & 100.0 \\
1000 & 100.0 & 100.0 & 100.0 & 100.0 & 100.0 \\
2000 & 100.0 & 100.0 & 100.0 & 100.0 & 100.0 \\
\hline \multicolumn{5}{c}{ Panel b: DGP 2} \\
\hline 250 & 68.9 & 40.2 & 26.6 & 19.3 & 16.5 \\
500 & 93.6 & 60.0 & 38.1 & 27.9 & 20.4 \\
1000 & 99.9 & 84.8 & 58.0 & 39.2 & 28.9 \\
2000 & 100.0 & 99.4 & 83.7 & 59.8 & 40.6 \\
\hline \multicolumn{5}{c}{ Panel c: DGP 3 } \\
\hline 250 & 35.5 & 36.0 & 32.9 & 31.9 & 31.9 \\
500 & 62.8 & 61.6 & 60.5 & 61.4 & 60.3 \\
1000 & 90.5 & 88.8 & 88.1 & 86.9 & 86.7 \\
2000 & 99.4 & 99.6 & 99.7 & 98.9 & 99.2 \\
\hline \hline
\end{tabular}

As expected, in Table 5 we observe that the goodness-of-fit test, $\widetilde{J}_{n}$, has the largest power for DGP 1 and it is not very powerful for DGP 2. It has reasonable power against DGP 3 mainly for samples of 1000 observations and above.

Table 5: Power of the $\widetilde{J}_{n}$-statistics $(n=13)$ under Parameter Uncertainty

\begin{tabular}{cccc}
\hline \hline$T$ & DGP 1 & DGP 2 & DGP 3 \\
\hline 250 & 99.1 & 12.4 & 19.7 \\
500 & 100.0 & 12.1 & 44.5 \\
1000 & 100.0 & 12.9 & 70.2 \\
2000 & 100.0 & 14.2 & 94.7 \\
\hline \hline
\end{tabular}

We find a similar message in Table 6 when we analyze the power of the $t$-statistics. The tests are the most powerful to detect DGP 1, the least powerful to detect DGP 2, and acceptable power against DGP 3 for samples of 1000 observations and above. There is a substantial drop in power for the $t_{11}$ test (90\% contour) for the cases of DGP 1 and DGP 3. This behavior is similar to that encountered in the univariate tests of González-Rivera, 
Senyuz, and Yoldas (2007). This is a result due to the specific density under the null. In the case of DGP 1, for some contour coverage levels the normal density and the Student-t are very similar. Hence it is very difficult for any test to discriminate the null from the alternative with respect to the coverage level of those contour planes. A similar argument applies to DGP 3 as well, since the GARCH structure in the conditional covariance matrix is associated with a non-normal unconditional density.

Table 6: Power of the t-statistics under Parameter Uncertainty

\begin{tabular}{|c|c|c|c|c|c|c|c|c|c|c|c|c|c|}
\hline$T$ & $l_{1}$ & $I_{2}$ & $t_{3}$ & $t_{4}$ & $t_{5}$ & $t_{6}$ & $t_{7}$ & $t_{8}$ & $t_{9}$ & $t_{10}$ & $t_{11}$ & $t_{12}$ & $t_{13}$ \\
\hline \multicolumn{14}{|c|}{ Panel a: DGP 1} \\
\hline 250 & & & & & & 9 & & 96.6 & & & & & .2 \\
\hline 500 & & & & & 0.0 & & & 100.0 & & & & & 98.5 \\
\hline 00 & & & .9 & & & & & & .0 & & & .7 & \\
\hline 00 & & & & & & & & & & & & 4.9 & 100 \\
\hline \multicolumn{14}{|c|}{ Panel b: DGP 2} \\
\hline 250 & & & & & & & & 11.0 & & & & & $3 .$. \\
\hline & & & & & & & & & & & & .9 & 3. \\
\hline & 1 & & 8 & & & & & & & & & .9 & 13. \\
\hline מo & 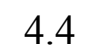 & 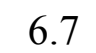 & 0 & & & 15.3 & 14.6 & 11.6 & & & & 2.3 & 14 \\
\hline \multicolumn{14}{|c|}{ Panel c: DGP 3} \\
\hline 250 & $\sigma$. & & & & & & & 18.7 & & & & & 17. \\
\hline- & 7.2 & 11. & 7.7 & 2 & 33.4 & & 41.5 & 41.1 & 2.6 & 15 & & 0.0 & 48 \\
\hline 000 & 8.1 & 20. & 31.4 & 46.3 & 58.6 & & 68.7 & 67.1 & & 32 & & 4.8 & 70 \\
\hline 00 & 3.5 & 35. & 56.8 & 77.7 & $86^{\circ}$ & 91.5 & 92.8 & 91.8 & 85. & 54 & 9. & 60.0 & 93 \\
\hline
\end{tabular}

\section{EMPIRICAL APPLICATIONS}

In this section we apply the proposed testing methodology to the generalized residuals of multivariate GARCH models fitted to U.S. stock return data. Our data set consists of daily excess returns on five size portfolios, i.e. portfolios sorted with respect to market capitalization in an increasing order. ${ }^{3}$ The sample period runs from January 2, 1996 to December 29, 2006, providing a total of 2770 observations. A plot of the data is provided in Figure-2.

\footnotetext{
${ }^{3}$ Data is obtained from Kenneth French's website: http://mba.tuck.dartmouth.edu/pages/faculty/ken.french. We are grateful to him for making this data publicly available.
} 
Figure-2: Daily Excess Returns on Five Size Portfolios (1/2/996-12/29/2006)

(From the smallest quintile portfolio to the largest quintile portfolio)
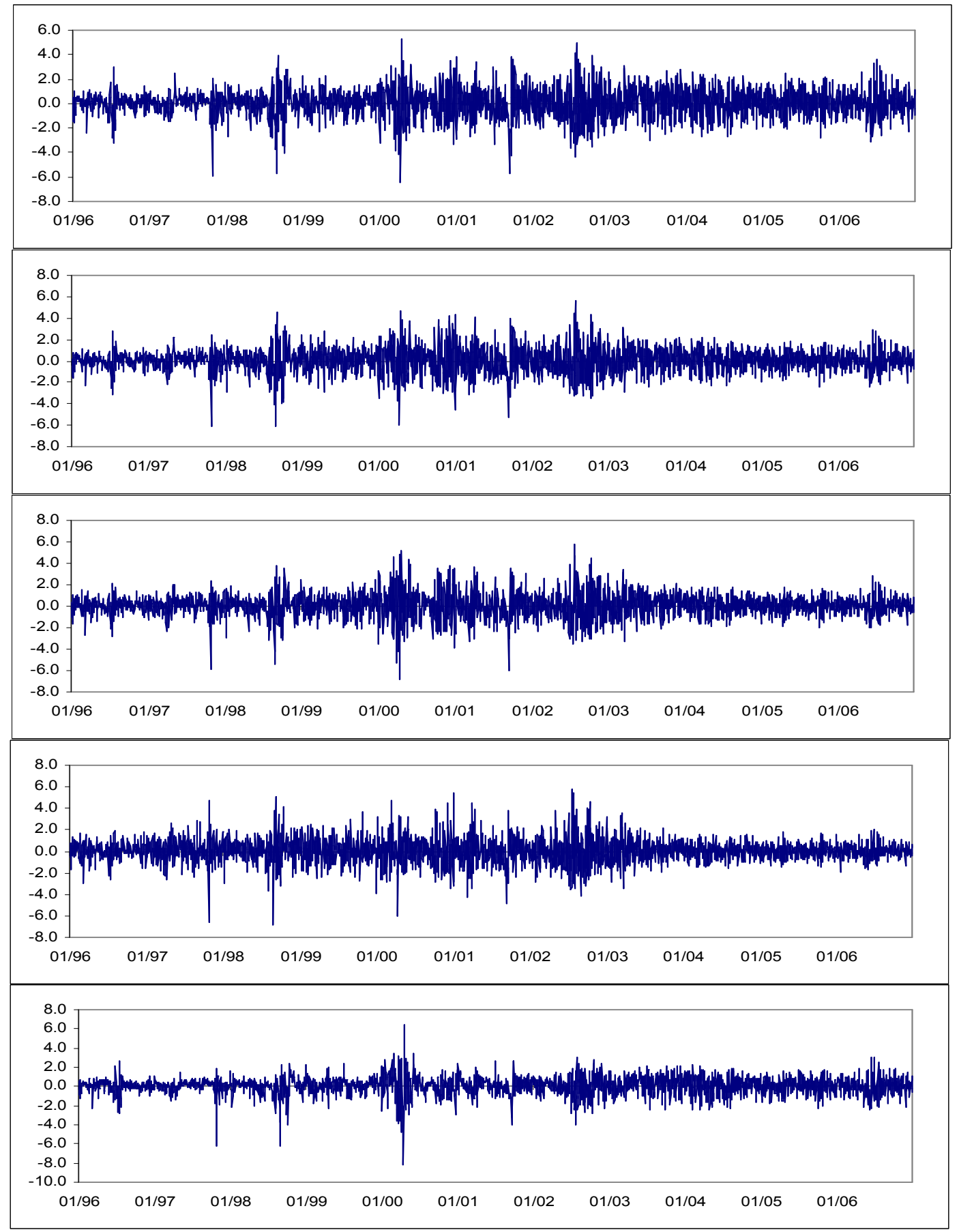
Since we are working with daily data we assume a constant conditional mean vector. In terms of the multivariate GARCH specifications, we consider two popular alternatives: the BEKK model of Engle and Kroner (1995) and the DCC model of Engle (2002). Define $u_{t}=y_{t}-\mu$ where $\mu$ is the constant conditional mean vector. Then the $\operatorname{BEKK}(1,1, K)$ specification for the conditional covariance matrix, $H_{t} \equiv E\left[u_{t} u_{t}^{\prime} \mid \mathfrak{J}_{t-1}\right]$, is given by

$$
H_{t}=C^{\prime} C+\sum_{j=1}^{K} A_{j}^{\prime} u_{t} u_{t-1}^{\prime} A_{j}+\sum_{j=1}^{K} G_{j}^{\prime} H_{t-1} G_{j}
$$

In our applications we set $K=1$ and use the scalar version of the model due to parsimony considerations where $A=\alpha I_{k}, A=\beta I_{k}$, and $\alpha$ and $\beta$ are scalars. We also use variance targeting to facilitate estimation, i.e. we set $C^{\prime} C=V-A^{\prime} V A-G^{\prime} V G$ where $V=E\left[u_{t} u_{t}^{\prime}\right]$, e.g. Ding and Engle (2001).

In the DCC specification, conditional variances and conditional correlations are modeled separately. Specifically, consider the following decomposition of the conditional covariance matrix: $H_{t}=D_{t} R_{t} D_{t}$ where $D_{t}=\operatorname{diag}\left\{h_{11, t}^{1 / 2}, \ldots, h_{k k, t}^{1 / 2}\right\}$, and each element of $D_{t}$ is modeled as an individual GARCH process. In our applications, we consider the standard GARCH $(1,1)$ process:

$$
h_{i i, t}=\omega_{i}+\alpha_{i} u_{i, t-1}^{2}+\beta_{i} h_{i i, t-1}, \quad j=1, \ldots, k
$$

Now define $z_{t}=D_{t}^{-1} u_{t}$, then $R_{t}=\operatorname{diag}\left\{Q_{t}\right\}^{-1} Q_{t} \operatorname{diag}\left\{Q_{t}\right\}^{-1}$ where

$$
Q_{t}=(1-\alpha-\beta) \bar{Q}+\alpha u_{t} u_{t-1}^{\prime}+\beta Q_{t-1}
$$

and $\bar{Q}=E\left[z_{t} z_{t-1}^{\prime}\right]$.

Under both BEKK and DCC specifications, we consider two alternative distributional assumptions that are most commonly used in empirical applications involving multivariate 
GARCH models: multivariate Normal and multivariate Student-t distributions. Under multivariate normality, the sample log-likelihood function, up to a constant, is given by

$$
L_{T}(\theta)=-\frac{1}{2} \sum_{t=1}^{T} \ln \left[\operatorname{det}\left(H_{t}\right)\right]-\frac{1}{2} \sum_{t=1}^{T} u_{t}^{\prime} H_{t} u_{t}
$$

In the case of the DCC model, a two-step estimation procedure is applicable under normality as one can write the total likelihood as the sum of two parts where the former depends on the individual GARCH parameters and the latter on the correlation parameters. Under this estimation strategy, consistency is still guaranteed to hold. For further details on two-step estimation in the DCC model, the interested reader is referred to Engle (2002), and Engle and Sheppard (2001). Under the assumption of multivariate Student-t distribution, we do not need to estimate the model with the corresponding likelihood since the estimates obtained under normality are consistent due to quasi maximum likelihood interpretation. Therefore, we obtain the standardized residual vectors under normality and then simply test the Student-t assumption on these residuals. ${ }^{4}$ One remaining issue in the case of Student-t distribution is the choice of the degrees of freedom. We follow Pesaran and Zaffaroni (2008) and obtain estimates of the degrees of freedom parameters for all series separately and then consider an average of the individual estimates for the distributional specification in the multivariate model.

The results are summarized in Figures 3 through 6 and Table 7. From the figures we observe that under both GARCH specifications, the $J_{n}^{l}$-statistics are highly statistically significant when multivariate normality is the maintained distributional assumption. The $J_{n}^{l}$-Statistics of the BEKK model are larger than those obtained under the DCC specification. Furthermore, there is an obvious pattern in the behavior of the statistics as a function of the lag order, especially under the BEKK specification. This indicates that the rejection is partly due to remaining dependence in the model residuals. When we switch to

\footnotetext{
${ }^{4}$ Note that in the specification of the multivariate Student-t distribution (see Section 2), the covariance matrix is already scaled to be an identity matrix, thus no re-scaling of residuals is necessary to implement the test, e.g. Harvey, Ruiz and Santana (1992).
} 
the multivariate Student-t distribution with 11 degrees of freedom, ${ }^{5}$ the $J_{n}^{l}$-statistics go down substantially under both multivariate GARCH specifications. Hence, we can argue that the distributional assumption plays a greater role in the rejection of both models under normality. The $J_{n}^{l}$-statistics are barely significant at $5 \%$ level for only a few lag values under the DCC specification coupled with multivariate Student-t distribution. However, under the BEKK specification, $J_{n}^{l}$-statistics are significant at early lags, even at $1 \%$ level. Table-10 reports individual $t$-statistics and the $\widetilde{J}_{n}$-statistics. Both types of test statistics indicate that normality is very strongly rejected under both GARCH specifications. Similar to the case of $J_{n}^{l}$-statistics, the results dramatically change when the distributional assumption is altered to multivariate Student-t. The DCC model produces better results with respect to both types of test statistics, but especially chi-squared test strongly supports the DCC specification compared to the BEKK model. Combining the information from all test statistics we can conclude that multivariate normality is a bad assumption to make regardless of the multivariate GARCH specification. Furthermore, the DCC model with multivariate Student-t distribution does a good job in terms of capturing dependence and producing a reasonable fit with respect to density functional form.

\footnotetext{
${ }^{5}$ This value is obtained by averaging individual degrees of freedom estimates obtained from individual GARCH models under Student-t density.
} 
Figure 3: $J_{13}^{l}$-statistics of BEKK Model under Multivariate Normal Distribution

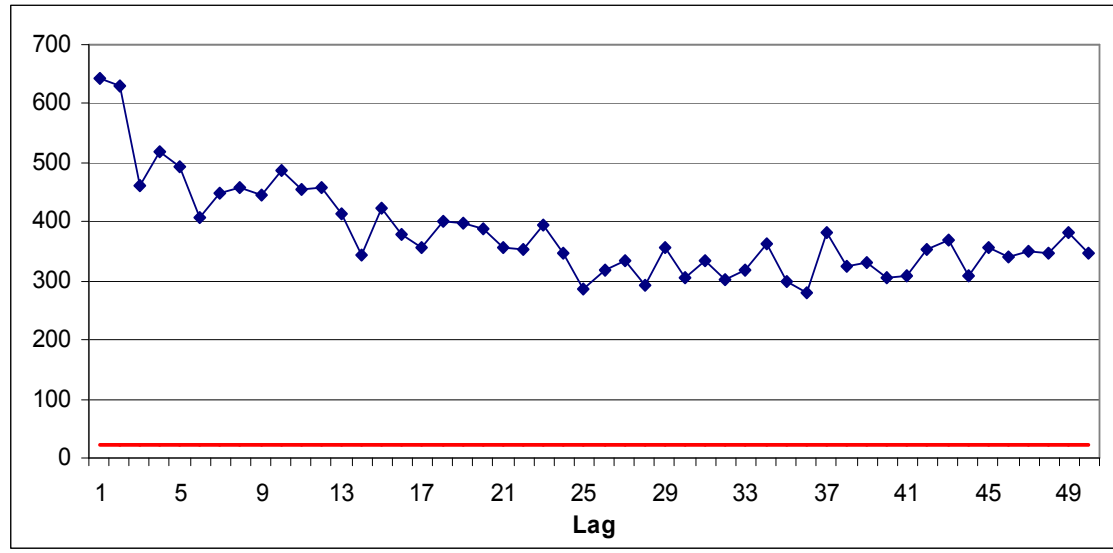

Figure 4: $J_{13}^{l}$-statistics of DCC Model under Multivariate Normal Distribution

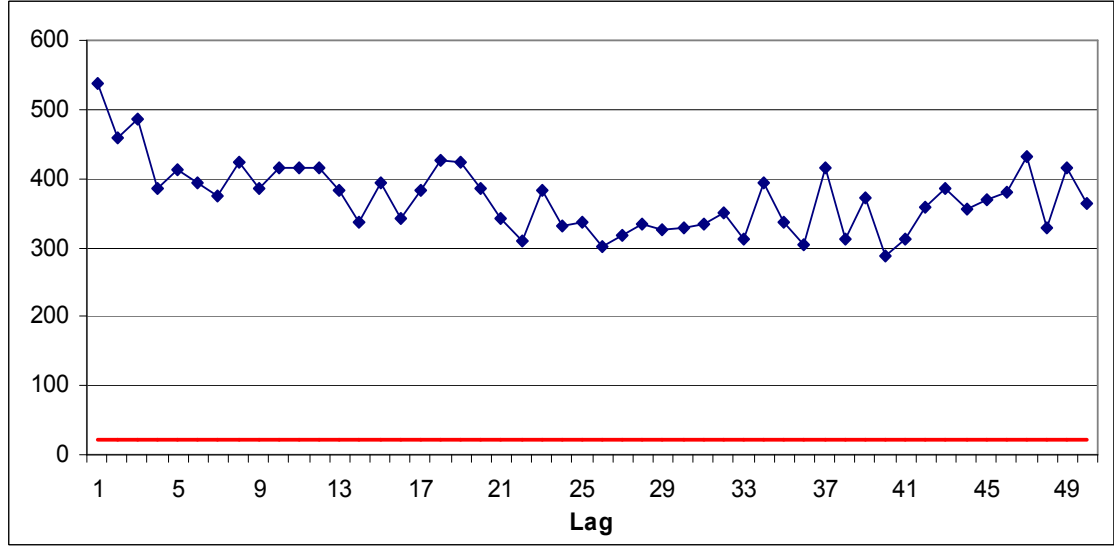

Figure 5: $J_{13}^{l}$-statistics of BEKK Model under Multivariate Student-t Distribution

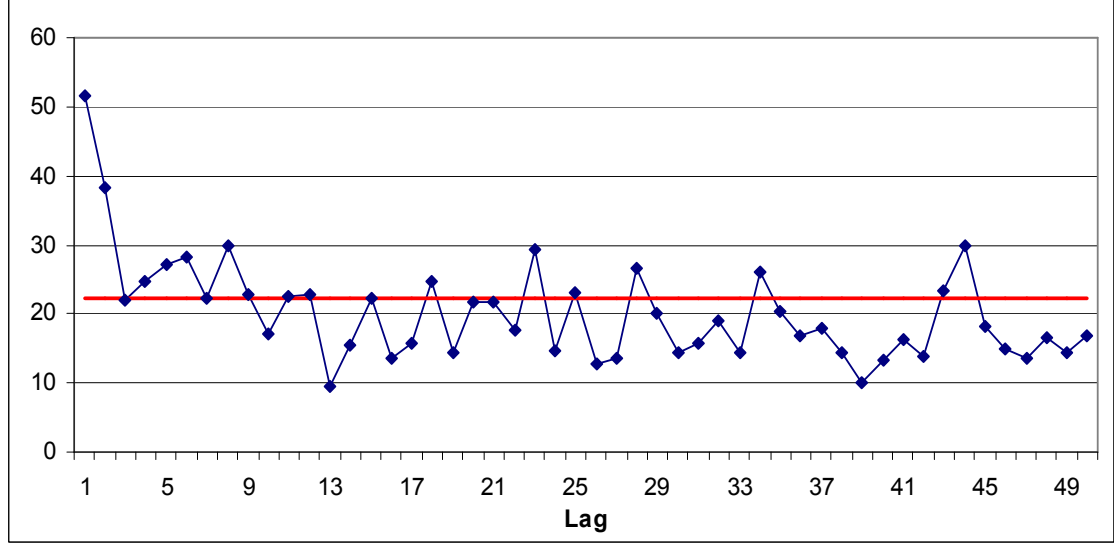


Figure 6: $J_{13}^{l}$-statistics of DCC Model under Multivariate Student-t Distribution

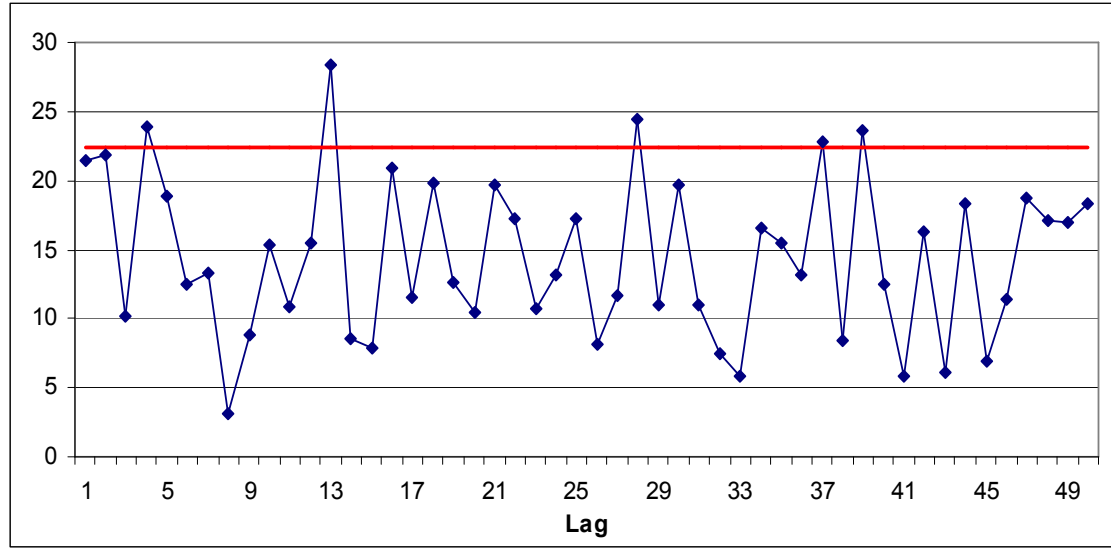

Table 7: Individual $t$ and $\widetilde{J}_{13}$-statistics for Estimated GARCH Models

\begin{tabular}{|c|c|c|c|c|}
\hline & $\begin{array}{c}\text { BEKK } \\
\text { Normal }\end{array}$ & $\begin{array}{c}\text { DCC } \\
\text { Normal }\end{array}$ & $\begin{array}{c}\text { BEKK } \\
\text { Student-t }\end{array}$ & $\begin{array}{c}\text { DCC } \\
\text { Student-t }\end{array}$ \\
\hline$t_{1}$ & -1.85 & -2.17 & 2.78 & 2.30 \\
\hline$t_{2}$ & -8.52 & -10.18 & -0.31 & -0.38 \\
\hline$t_{3}$ & -9.97 & -12.26 & 1.00 & -0.64 \\
\hline$t_{4}$ & -9.37 & -11.22 & 0.84 & -0.10 \\
\hline$t_{5}$ & -10.34 & -11.81 & 2.47 & 0.18 \\
\hline$t_{6}$ & -11.54 & -10.95 & 1.13 & 0.95 \\
\hline$t_{7}$ & -9.28 & -10.03 & 0.09 & 0.50 \\
\hline$t_{8}$ & -6.85 & -7.19 & 0.25 & 0.59 \\
\hline$t_{9}$ & -2.74 & -5.70 & 0.92 & -0.32 \\
\hline$t_{10}$ & 0.24 & -1.52 & 0.66 & -0.89 \\
\hline$t_{11}$ & 5.39 & 2.17 & 0.08 & -3.51 \\
\hline$t_{12}$ & 8.23 & 5.58 & 1.00 & -1.30 \\
\hline$t_{13}$ & 12.18 & 12.50 & 1.26 & 0.74 \\
\hline$\widetilde{J}_{13}$ & 351.47 & 388.54 & 30.07 & 24.35 \\
\hline
\end{tabular}




\section{CONCLUDING REMARKS}

Motivated by the relative scarcity of tests for dynamic specification and density functional form in multivariate time series models, we proposed a new battery of tests based on the concept of "autocontour" introduced by González-Rivera, Senyuz, and Yoldas (2007) for univariate processes. We developed $t$-tests based on a single autocontour and also more powerful chi-squared tests based on multiple autocontours, which have standard asymptotic distributions. We also developed a second type of chi-squared test statistic, which is informative as a goodness-of-fit test when combined with the first type of chisquared test. Monte-Carlo simulations indicate that the tests have good size and power against dynamic misspecification and deviations from the hypothesized density. We applied our methodology to multivariate GARCH models and showed that the DCC specification of Engle (2002) coupled with a multivariate Student-t distribution provides a fine model for multivariate time dependence in a relative large system of stock returns. 


\section{REFERENCES}

Bai, J. and Z. Chen (2008), Testing Multivariate Distributions in GARCH Models, Journal of Econometrics, 143, 19-36.

Bai J. (2003) Testing Parametric Conditional Distributions of Dynamic Models, Review of Economics and Statistics, 85, 532-549.

Bauwens, L., S. Laurent, and J.V.K. Rombouts (2006), Multivariate GARCH Models: A Survey, Journal of Applied Econometrics, 21, 79-109.

Ding, $Z$ and Engle, R.F. (2001), Large Scale Conditional Covariance Modeling, Estimation and Testing, Academia Economic Papers, 29, 157-184.

Duchesne, P. and S. Lalancette (2003), On Testing for Multivariate ARCH Effects in Vector Time Series Models, La Revue Canadienne de Statistique, 31, 275-292.

Engle, R.F. (2002), Dynamic Conditional Correlation-A simple Class of Multivariate GARCH Models, Journal of Business and Economic Statistics, 20, 339-350.

Engle, R.F. and K. Sheppard (2001), Theoretical and Empirical Properties of Dynamic Conditional Correlation Multivariate GARCH, Мimeo, UC San Diego.

Engle, R.F. and F.K. Kroner (1995), Multivariate Simultaneous Generalized GARCH, Econometric Theory, 11, 122-150.

González-Rivera, G. Senyuz, Z. and E. Yoldas (2007), Autocontours: Dynamic Specification Testing, Mimeo, UC Riverside.

Harvey, A., Ruiz, E., and E. Sentana (1992), Unobservable Component Time Series Models with ARCH Disturbances, Journal of Econometrics, 52, 129-158.

Kalliovirta, L. (2007), Quantile Residuals for Multivariate Models, Mimeo, University of Helsinki.

Ling, S. and W.K. Li (1997), Diagnostic Checking of Nonlinear Multivariate Time Series with Multivariate ARCH Errors, Journal of Time Series Analysis, 18, 447-464.

Pesaran M.H. and P. Zaffaroni (2008), Model Averaging in Risk Management with an Application to Futures Markets, CESifo Working Paper Series No. 1358; IEPR Working Paper No. 04.3.

Tse, Y.K. (2002), Residual-based Diagnostics for Conditional Heteroscedasticity Models, Econometrics Journal 5, 358-373.

Tse, Y.K. and A.K.C. Tsui (1999), A Note on Diagnosing Multivariate Conditional Heteroscedasticity Models, Journal of Time Series Analysis, 20, 679-691. 